\title{
The Number of Line-Convex Directed Polyominoes Having the Same Orthogonal Projections
}

\author{
Péter Balázs \\ Department of Computer Algorithms and Artificial Intelligence \\ University of Szeged \\ Árpád tér 2, H-6720 Szeged, Hungary \\ pbalazs@inf.u-szeged.hu
}

\begin{abstract}
The number of line-convex directed polyominoes with given horizontal and vertical projections is studied. It is proven that diagonally convex directed polyominoes are uniquely determined by their orthogonal projections. The proof of this result is algorithmical. As a counterpart, we show that ambiguity can be exponential if antidiagonal convexity is assumed about the polyomino. Then, the results are generalised to polyominoes having convexity property along arbitrary lines.
\end{abstract}

Keywords: Discrete tomography; line-convex directed polyomino; reconstruction from projections.

\section{Introduction}

The reconstruction of two-dimensional discrete sets (the finite subsets of $\mathbb{Z}^{2}$ ) from their projections is a frequently studied area of discrete tomography [1] and it has its applications in pattern recognition, image processing, electron microscopy, and radiology $[2,3,4,5,6]$. For practical reasons the number of projections used in the reconstruction is small (usually two or four). Thus, in certain cases the number of discrete sets having the same projections can be extremely large leading to a reconstructed set which is possibly quite dissimilar to the original one. A commonly used technique to reduce the number of solutions is to suppose having some a priori information of the set to be reconstructed such as convexity, connectedness and directedness. Some properties imposed on the set to be reconstructed completely eliminate ambiguity and make it possible to develope efficient reconstruction algorithms [7,8,9]. However, there are classes of discrete sets where ambiguity is only partially eliminated making the reconstruction of the set very difficult [10,11. In this paper we investigate the problem of ambiguity when the set to be reconstructed must be line-convex, connected, and directed.

This contribution is structured as follows. First, the necessary definitions are introduced in Section 2, In Section 3 we study diagonally convex directed polyominoes. We give a uniqueness result for this class and derive an algorithm for 
reconstructing the uniquely determined polyomino from its horizontal and vertical projections. In Section 4 we show that ambiguity can remain very high when the polyominoes to be reconstructed are antidiagonally convex. In Section 5 we consider the possibility to adapt the results of Sections 3 and 4 to polyominoes that are convex along an arbitrary set of directions. Finally, in Section 6 we conclude our results.

\section{Preliminaries}

The finite subsets of $\mathbb{Z}^{2}$ defined up to translation are called discrete sets. A discrete set $F$ can be represented by a set of unitary cells or by a binary matrix $\hat{F}=\left(\hat{f}_{i j}\right)_{m \times n}$ where $\hat{f}_{i j}=1$ if and only if $(i, j) \in F$. To stay consistent we assume that the vertical axis of the two-dimensional integer lattice is directed top down (see Fig. 1). The horizontal and vertical projections of $F$ are the vectors $\mathcal{H}(F)=H=\left(h_{1}, \ldots, h_{m}\right)$, and $\mathcal{V}(F)=V=\left(v_{1}, \ldots, v_{n}\right)$, respectively, where

$$
h_{i}=\sum_{j=1}^{n} \hat{f}_{i j} \quad(i=1, \ldots, m) \quad \text { and } \quad v_{j}=\sum_{i=1}^{m} \hat{f}_{i j} \quad(j=1, \ldots, n) .
$$

Not any pair of vectors are the projections of some discrete set. In the following we suppose that $H \in \mathbb{N}_{0}^{m}$ and $V \in \mathbb{N}_{0}^{n}$ are compatible which means that they satisfy the following conditions

(i) $h_{i} \leq n$, for $1 \leq i \leq m$, and $v_{j} \leq m$, for $1 \leq j \leq n$;

(ii) $\sum_{i=1}^{m} h_{i}=\sum_{j=1}^{n} v_{j}$, i.e., the two vectors have the same total sums.

Two points $P=\left(p_{1}, p_{2}\right)$ and $Q=\left(q_{1}, q_{2}\right)$ in $\mathbb{Z}^{2}$ are said to be 4 -adjacent if $\left|p_{1}-q_{1}\right|+\left|p_{2}-q_{2}\right|=1$. The sequence of distinct points $P_{0}, \ldots, P_{k}$ is a 4 -path from point $P_{0}$ to point $P_{k}$ in a discrete set $F$ if each point of the sequence is in $F$ and $P_{l}$ is 4 -adjacent to $P_{l-1}$ for each $l=1, \ldots, k$. A discrete set $F$ is 4connected if for any two points in $F$ there is a 4-path between them. Such a set is also called as polyomino. A 4-path in a discrete set $F$ is a northeast path (or shortly, $N E$-path) from point $P_{0}$ to point $P_{k}$ if each point $P_{l}$ of the path is in north or east to $P_{l-1}$ for each $l=1, \ldots, k$. The discrete set $F$ is directed if there is a NE-path in $F$ from point $(m, 1)$ to any other point of $F$.

Given two integers $a$ and $b$ such that they are coprimes and $(a, b) \neq(0,0)$ we define the $k$ th line of the discrete rectangle $R=\{1, \ldots, m\} \times\{1, \ldots, n\}$ parallel to the vector $(a, b)$ by

$$
S_{k}^{(a, b)}=\{(i, j) \in R \mid b i-a j=k\} .
$$

Throughout this paper, without loss of generality, we will assume that $b \geq 0$ and $b=0$ if and only if $a=1$. The discrete set $F$ is line-convex along the direction $(a, b)$ if for every $k \in \mathbb{Z}$ and $\left(i_{1}, j_{1}\right),\left(i_{2}, j_{2}\right) \in S_{k}^{(a, b)} \cap F$ the discrete line segment with the endpoints $\left(i_{1}, j_{1}\right)$ and $\left(i_{2}, j_{2}\right)$ is in $F$, i.e., if $(i, j) \in S_{k}^{(a, b)}$ such that $(i, j)=\left(i_{1}+t\left(i_{2}-i_{1}\right), j_{1}+t\left(j_{2}-j_{1}\right)\right)$ where $t \in[0,1]$ then $(i, j) \in F$. In particular, the discrete set is 


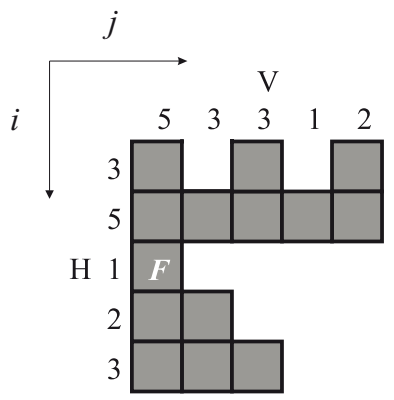

$$
\hat{F}=\left(\begin{array}{lllll}
1 & 0 & 1 & 0 & 1 \\
1 & 1 & 1 & 1 & 1 \\
1 & 0 & 0 & 0 & 0 \\
1 & 1 & 0 & 0 & 0 \\
1 & 1 & 1 & 0 & 0
\end{array}\right)
$$

Fig. 1. A diagonally convex directed polyomino $F$, and the correspondig binary matrix $\hat{F}$. The projections of the polyomino are the vectors $H$ and $V$.

- horizontally convex if it is line-convex along the direction $(0,1)$,

- vertically convex if it is line-convex along the direction $(1,0)$,

- diagonally convex if it is line-convex along the direction $(1,1)$,

- antidiagonally convex if it is line-convex along the direction $(-1,1)$.

For example, Fig. 1 shows a diagonally convex directed polyomino, which is vertically, horizontally, and antidiagonally non-convex.

\section{Diagonally Convex Directed Polyominoes}

Polyominoes are widely used in physics and chemistry for modelling and they have long been studied by mathematicians and computer scientists (see [11, 12, 13 and the references given there). Concerning polyominoes with some lineconvexity properties some important results are already known. In [1] the authors studied the number of (horizontally and vertically) convex polyominoes reconstructible from their orthogonal projections and showed that in this class ambiguity can be very high. Moreover, in [12] a method is given to enumerate diagonally convex directed polyominoes according to several parameters (sources, diagonals, horizontal and vertical edges, etc.). Recently, in [14 the author stressed the importance of finding classes of polyominoes where the reconstruction from two projections can be solved uniquely in polynomial time. The only class known so far satisfying this condition was investigated in [8, 9 . The results given there can be summarized in the following.

Theorem 1. Every horizontally or vertically convex directed polyomino can be reconstructed from its horizontal and vertical projections uniquely in $O(m n)$ time.

In this section we show that the class of diagonally convex directed polyominoes (let us denote this class by $\mathcal{D C} \mathcal{C}_{4}$ ) also satisfies this condition. As an inmediate consequence of the directedness and 4-connectedness we can say 
Lemma 1. Let $D \in \mathcal{D C D}_{4}$ with $\mathcal{H}(D)=\left(h_{1}, \ldots, h_{m}\right)$ and $\mathcal{V}(D)=\left(v_{1}, \ldots, v_{n}\right)$. Then, $(m, j) \in D$ if and only if $1 \leq j \leq h_{m}$, and $(i, 1) \in D$ if and only if $m-v_{1}<i \leq m$.

With the aid of Lemma 1 a subset $F$ of the polyomino $D$ to be reconstructed can easily be found. On the basis of the following lemma the remaining elements of $D$ are determined by the set $F$.

Lemma 2. Let $D \in \mathcal{D C D}_{4}, F \subset D$ and $(i, j) \in\{1, \ldots, m-1\} \times\{2, \ldots, n\}$ be $a$ position such that for every $\left(i^{\prime}, j^{\prime}\right) \neq(i, j)$ if $i^{\prime} \geq i$ and $j^{\prime} \leq j$ then $\left(i^{\prime}, j^{\prime}\right) \in D \leftrightarrow$ $\left(i^{\prime}, j^{\prime}\right) \in F$. Then, $(i, j) \in D$ if and only if $\sum_{t=i+1}^{n} \hat{f}_{t j}<v_{j}$ and $\sum_{t=1}^{j-1} \hat{f}_{i t}<h_{i}$.

Proof. Let $(i, j)$ be a position satisfying the conditions of the lemma. The necessary part is trivial since $i^{\prime} \geq i, j^{\prime} \leq j$ and $\left(i^{\prime}, j^{\prime}\right) \neq(i, j)$ implies $\left(i^{\prime}, j^{\prime}\right) \in D \leftrightarrow$ $\left(i^{\prime}, j^{\prime}\right) \in F$ and so the inequalities $\sum_{t=i+1}^{n} \hat{f}_{t j}<v_{j}$ and $\sum_{t=1}^{j-1} \hat{f}_{i t}<h_{i}$ must hold. To prove the sufficient part assume that $\sum_{t=i+1}^{n} \hat{f}_{t j}<v_{j}$ and $\sum_{t=1}^{j-1} \hat{f}_{i t}<h_{i}$ and contrary $(i, j) \notin D$, i.e., $\hat{d}_{i j}=0$. If $i=1$ then the contradiction follows from $\sum_{t=i+1}^{n} \hat{f}_{t j}<v_{j}$ and the fact that $\left(i^{\prime}, j\right) \in F \leftrightarrow\left(i^{\prime}, j\right) \in D$ holds for every position $\left(i^{\prime}, j\right)$ if $i^{\prime}>1$. Similarly, if $j=n$ then the contradiction follows from $\sum_{t=1}^{j-1} \hat{f}_{i t}<h_{i}$ and the fact that $\left(i, j^{\prime}\right) \in F \leftrightarrow\left(i, j^{\prime}\right) \in D$ holds for every position $\left(i, j^{\prime}\right)$ if $j^{\prime}<n$. In any other cases, since the conditions of the lemma hold, there exist $i^{\prime \prime}<i$ and $j^{\prime \prime}>j$ for which $\hat{d}_{i^{\prime \prime}, j}=\hat{d}_{i, j^{\prime \prime}}=1$. Since $D$ is directed there is a NE-path from $(m, 1)$ to $\left(i^{\prime \prime}, j\right)$ such that for every point $\left(c_{1}, c_{2}\right)$ of this path $c_{2} \leq j$ holds. Therefore the diagonal $S_{i-j}^{(1,1)}$ contains at least one point of $D$, say $\left(i_{1}, j_{1}\right)$ for which $j_{1}<j$. Similarily, we get that there is a NE-path from $(m, 1)$ to $\left(i, j^{\prime \prime}\right)$ and therefore the diagonal $S_{i-j}^{(1,1)}$ contains at least one point of $D$, say $\left(i_{2}, j_{2}\right)$ for which $j_{2}>j$. We get $\hat{d}_{i_{1}, j_{1}}=1, \hat{d}_{i j}=0$ and $\hat{d}_{i_{2}, j_{2}}=1$ with $j_{1}<j<j_{2}$ which contradicts the diagonal convexity (see Fig. 2).

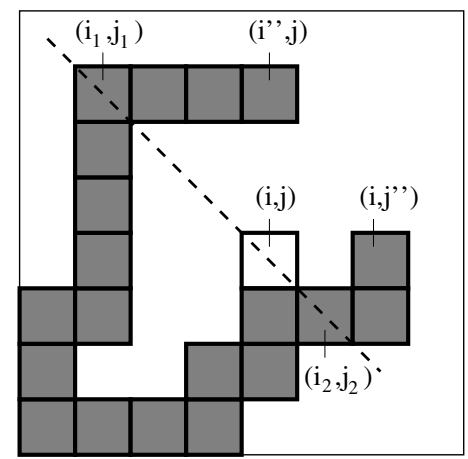

Fig. 2. Proof of Lemma 2 
The following theorem states that if we assume that the directed polyomino to be reconstructed is diagonally convex then ambiguity can be completely eliminated.

Theorem 2. Let $H \in \mathbb{N}^{m}$ and $V \in \mathbb{N}^{n}$. In the class $\mathcal{D C D} \mathcal{D}_{4}$ there is at most one polyomino $P$ such that $\mathcal{H}(P)=H$ and $\mathcal{V}(P)=V$.

Proof. On the basis of Lemma 1 the first column and the last row of $P$ are uniquely determined by $v_{1}$ and $h_{m}$, repsectively, i.e., a subset $F$ of the polyomino $P$ (consisting of all the positions of the last row and first column of $P$ ) can be found. Then, for the position $(m-1,2)$ the conditions of Lemma 2 hold. Therefore on the basis of Lemma 2 it can be decided whether the position $(m-1,2)$ belongs to $P$ and if so then we set $F=F \cup\{(m-1,2)\}$. Taking each position bottom up left to right $F$ always satisfies the conditions of Lemma 2 and so the above method can be repeated. If $H$ and $V$ are the projections of a diagonally convex polyomino then in the end we get $F=P$. Uniqueness follows from the construction.

The proof of Theorem 2 is constructive, i.e., an algorithm similar to the one in 9] can also be described to reconstruct the possibly existing polyomino of the class $\mathcal{D C D}_{4}$ with given horizontal and vertical projections.

\section{Algorithm DCD4}

Input: Two compatible vectors, $H \in \mathbb{N}^{m}$ and $V \in \mathbb{N}^{n}$.

Output: The binary matrix $\hat{F}$ representing the uniquely determined polyomino of $\mathcal{D C D} D_{4}$ having projections $H$ and $V$ (if there is such a solution).

Step $1 \hat{F}:=(0)_{m \times n} ; H^{\prime}:=H ; V^{\prime}:=V$;

Step 2 for $i:=m-v_{1}+1, \ldots, m\left\{\hat{f}_{i 1}:=1 ; h_{i}^{\prime}--;\right\}$

for $j:=1, \ldots, h_{m}\left\{\hat{f}_{m j}:=1 ; v_{j}^{\prime}--;\right\}$

Step 3 for $i:=m-1, \ldots, 1$

$$
\begin{aligned}
& \text { for } j:=2, \ldots, n \\
& \quad \text { if }\left(h_{i}^{\prime}>0 \text { and } v_{j}^{\prime}>0\right) \text { then }\left\{\hat{f}_{i j}:=1 ; h_{i}^{\prime}--; v_{j}^{\prime}--;\right\}
\end{aligned}
$$

Step 4 if $(\mathcal{H}(F) \neq H$ or $\mathcal{V}(F) \neq V$ or $F$ is not a diagonally convex polyomino) then exit(no solution) else return $\hat{F}$;

This algorithm works as follows. Step 1 is for the initialization of the matrix $\hat{F}$ and the auxiliary vectors $H^{\prime}$ and $V^{\prime}$. In Step 2 a subset $F$ of the polyomino to be reconstructed is defined on the basis of Lemma 1. Then, in each iteration of Step 3 we check whether the conditions of Lemma 2 hold and if so then we update the matrix $\hat{F}$ and the vectors $H^{\prime}$ and $V^{\prime}$. Due to the vectors $H^{\prime}$ and $V^{\prime}$ Step 3 runs in $O(m n)$ time. Finally, in Step 4 we check whether the reconstructed set is a diagonally convex polyomino and has the given projections $H$ and $V$ which can also be done in $O(m n)$ time. Summarizing this we can say

Corollary 1. Let $H \in \mathbb{N}^{m}$ and $V \in \mathbb{N}^{n}$. If there is a polyomino $P \in \mathcal{D C D} \mathcal{D}_{4}$ such that $\mathcal{H}(P)=H$ and $\mathcal{V}(P)=V$ then Algorithm DCD4 reconstructs it in $O(m n)$ time. 


\section{Antidiagonally Convex Directed Polyominoes}

In this section we show that there is a drastic change in the number of directed polyominoes if instead of diagonal convexity it is assumed that the polyominoes to be reconstructed are antidiagonally convex. We will use the concept of switching component of a binary matrix $\hat{F}$ which is a submatrix of $\hat{F}$ of the form

$$
\left(\begin{array}{ll}
0 & 1 \\
1 & 0
\end{array}\right) \text { or }\left(\begin{array}{ll}
1 & 0 \\
0 & 1
\end{array}\right) \text {. }
$$

Interchanging $0 \mathrm{~s}$ and $1 \mathrm{~s}$ in a switching component the horizontal and vertical projections of $F$ do not change [15. Let us denote the class of antidiagonally convex directed polyominoes by $\mathcal{A C D}_{4}$. The following theorem shows that assuming antidiagonal convexity on the directed polyomino having given horizontal and vertical projections does not eliminate ambiguity.

Theorem 3. In the class $\mathcal{A C D}_{4}$ there can be exponentially large number of polyominoes with the same horizontal and vertical projections.

Proof. We show that for any $k \in \mathbb{N}$ there are at least $2^{k}$ number of discrete sets of size $(6 k-1) \times 3$ in the class $\mathcal{A C D}_{4}$ with the same horizontal and vertical projections. Let

$$
\hat{B}=\left(\begin{array}{lll}
1 & 1 & 0
\end{array}\right), \quad \hat{F}_{1}=\left(\begin{array}{ccc}
1 & 1 & 0 \\
1 & 1 & 1 \\
1 & 1 & 0 \\
1 & 0 & 1 \\
1 & 1 & 1
\end{array}\right), \quad \text { and } \quad \hat{F}_{k}=\left(\begin{array}{c}
\hat{F}_{1} \\
\hat{B} \\
\hat{F}_{k-1}
\end{array}\right) \quad \text { for } \quad k>1
$$

For a given $k \in \mathbb{N}$ and for any $l \in \mathbb{N}(1 \leq l \leq k)$ we will refer to the submatrix of $\hat{F}_{k}$ consisting of the rows $6(l-1)+i \quad(i=1, \ldots, 5)$ as the lth level of $\hat{F}_{k}$ and to the submatrix $\hat{B}$ in the row $6 l$ as the lth bridge of $\hat{F}_{k}$ (omitting the case $k=l)$. For any $l$ the positions $(6(l-1)+4,2),(6(l-1)+4,3),(6(l-1)+1,2)$, and $(6(l-1)+1,3)$ form a switching component in $\hat{F}_{k}$ and we will refer to it as the lth switching component of $\hat{F}_{k}$. Let $\hat{F}_{1}^{\prime}$ be the binary matrix that we get by interchanging the $0 \mathrm{~s}$ and $1 \mathrm{~s}$ in the first switching component of $\hat{F}_{1}$, i.e.,

$$
\hat{F}_{1}^{\prime}=\left(\begin{array}{ccc}
1 & 0 & 1 \\
1 & 1 & 1 \\
1 & 1 & 0 \\
1 & 1 & 0 \\
1 & 1 & 1
\end{array}\right)
$$

Clearly, $F_{1}, F_{1}^{\prime} \in \mathcal{A C D}_{4}$ and so the theorem holds for the case $k=1$. For the case $k>1$ let $\hat{F}_{k}^{S}$ where $S=\left\{s_{1}, \ldots, s_{n}\right\} \subseteq\{1, \ldots, k\}(n \leq k)$ denote the binary matrix that we get from $\hat{F}_{k}$ by switching the $s_{1}$ th, $\ldots, s_{n}$ th switching components. Note, that from the viewpoint of directedness, 4-connectedness and antidiagonal convexity the $l$ th bridge effects only on the $(l+1)$-th and $l$ th levels 
and vice versa (if they exist). Then, in order to prove that $F_{k}^{S} \in \mathcal{A C D}_{4}$ for any $k \in \mathbb{N}$ and $S \subseteq\{1, \ldots, k\}$ it is sufficient to study the submatrices of $\hat{F}_{k}^{S}$ consisting of the $l$ th level and the $l$ th bridge and the $l$ th bridge and the $(l+1)$-th level. These matrices can only be of the form $\left(\begin{array}{c}\hat{B} \\ \hat{F}_{1}\end{array}\right),\left(\begin{array}{c}\hat{F}_{1} \\ \hat{B}\end{array}\right),\left(\begin{array}{c}\hat{B} \\ \hat{F}_{1}^{\prime}\end{array}\right)$, or $\left(\begin{array}{c}\hat{F}_{1}^{\prime} \\ \hat{B}\end{array}\right)$. It can be shown directly that the four sets represented by these matrices are antidiagonally convex. For a given $k S$ can be any subset of $\{1, \ldots, k\}$ therefore the number of solutions with the same projections is at least $2^{k}$ and so the theorem is proven (see Fig. [ for the case $k=2$ ).

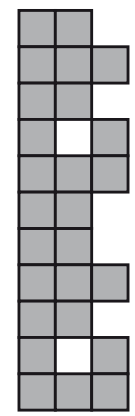

(a)

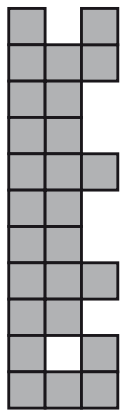

(b)

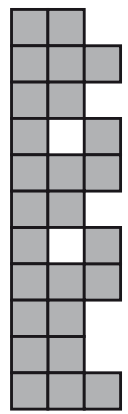

(c)

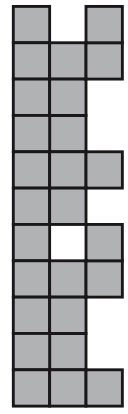

(d)

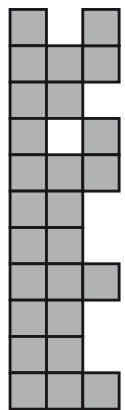

(e)

Fig. 3. Exponentially large number of discrete sets of $\mathcal{A C D}_{4}$ with the same horizontal and vertical projections. (a)-(d) Proof of Theorem 3 for the case $k=2$. The sets left to right are $F_{2}^{\{\}}, F_{2}^{\{1\}}, F_{2}^{\{2\}}$, and $F_{2}^{\{1,2\}}$, respectively. (e) One more set with the same projections.

Remark 1. The bound $2^{k}$ in the proof of Theorem 3 is not tight. See, for example, the discrete set in Fig. 3 e.

As a consequence of this theorem we get

Corollary 2. If there is an algorithm that reconstructs all the discrete sets of $\mathcal{A C D}_{4}$ with the horizontal and vertical projections $H$ and $V$, respectively, then there are some pair of vectors $H$ and $V$ for which the time complexity of the algorithm is not polynominal.

\section{Generalisation to Arbitrary Line-Convexity}

From Sections 3 and 4 it is clear that the direction of convexity has an important role in whether or not ambiguity can be eliminated. In this section we study in more detail how the direction of convexity effects on the number of directed polyominoes. First, note that without further modification Theorem 2 can be 
stated in a more general way to polyominoes that are line-convex along the direction $(a, b)$ if $a>0$ holds. Moreover, the construction given in the proof of Theorem 3 can be adapted to polyominoes that are line-convex along any direction $(a, b)$ for which $a<0$ in the following way.

Theorem 4. Let $\mathcal{C}_{(a, b)}$ be the class of polyominoes that are line-convex along the direction $(a, b)$ such that $a<0$. Then, there can be exponentially large number of polyominoes of $\mathcal{C}_{(a, b)}$ with the same horizontal and vertical projections.

Proof. (Sketch.) Assume that a direction $(a, b)$ is given with $a<0$. We will give a construction similar to the one used in the proof of Theorem 3 First, note that for each $k \geq 1$ the martices of (44) have only 3 columns therefore they are also line-convex along the direction $(a, b)$ if $b \geq 2$. Moreover, $a \neq 1$ implies $b \neq 0$. Thus, the theorem holds for $b \neq 1$. Consider $b=1$ and construct the bridge $\hat{B}^{(a)}$ of size $(-a) \times 3$ and the matrix $\hat{F}_{1}^{(a)}$ of size $(4-a) \times 3$ from the matrices given in (4) in the following way

$$
\hat{B}^{(a)}=\left(\begin{array}{ccc}
1 & 1 & 0 \\
& \vdots & \\
1 & 1 & 0
\end{array}\right), \quad \hat{F}_{1}^{(a)}=\left(\begin{array}{ccc}
1 & 1 & 0 \\
1 & 1 & 1 \\
& \hat{B}^{(a)} & \\
1 & 0 & 1 \\
1 & 1 & 1
\end{array}\right) .
$$

Notice that $\hat{B}^{(-1)}=\hat{B}$ and so $\hat{F}_{1}^{(-1)}=\hat{F}_{1}$. Then, for each $a<0$ the proof can be finished similarly as the proof of Theorem 3 .

Now, we can state the main result of our paper.

Theorem 5. Let $\mathcal{D}=\left\{\left(a_{i}, b_{i}\right) \mid i=1, \ldots, l\right\}$ be a finite set of directions and $P$ be a polyomino line-convex along every directions of $\mathcal{D}$. Then, $P$ is uniquely determined by its horizontal and vertical projections and it can be reconstructed in $O(m n)$ time if there exist a direction $(a, b) \in \mathcal{D}$ with $a \geq 0$. If $a_{i}<0$ for all directions $\left(a_{i}, b_{i}\right) \in \mathcal{D}(i=1, \ldots, l)$ then there can be exponentially large number of polyominoes that are line-convex along the directions of $\mathcal{D}$ and have the same horizontal and vertical projections as $P$.

Proof. It follows from Theorem 1 and the discussion of the above paragraph. The only non-trivial statement is the case if $a_{i}<0$ for all directions $\left(a_{i}, b_{i}\right) \in \mathcal{D}$ $(i=1, \ldots, l)$. In this situation we can have two cases. If there is at least one direction $\left(a_{j}, b_{j}\right)$ such that $b_{j}=1$ then we have to to apply the construction of (6) with the argument $a=\min \left\{a_{i} \mid b_{i}=1,\left(a_{i}, b_{i}\right) \in \mathcal{D}\right\}$. Otherwise, (that is, if $b_{i} \neq 1$ for all $\left.i=1, \ldots, l\right)$ we can simply use the construction of (4).

Remark 2. Theorem 5 can be extended to any infinte set $\mathcal{D}$ of directions except the case if $b_{i} \neq 1$ for all $\left(a_{i}, b_{i}\right) \in \mathcal{D}$. In this latter case the integer $\min \left\{a_{i} \mid b_{i}=\right.$ $\left.1,\left(a_{i}, b_{i}\right) \in \mathcal{D}\right\}$ does not exist. 


\section{Conclusions}

We have studied the problem of ambiguity in classes of directed discrete sets. We have shown that in the class of diagonally convex directed polyominoes the horizontal and vertical projections uniquely determine the polyomino and this polyomino can be reconstructed in $O(m n)$ time. However, assuming antidiagonal convexity about the polyomino the number of solutions with the same horizontal and vertical projections can be extremely large. Then, the results were generalised to polyominoes having arbitrary line-convexity. It is an open question whether in the classes where non-uniqueness holds a reconstruction algorithm can be given to find a solution in polynomial time. Another interesting question is if it is possible to generalise Theorem 5 to arbitrary infinite set of directions, too.

\section{References}

1. Herman, G.T., Kuba, A. (eds.): Discrete Tomography: Foundations, Algorithms and Applications. Birkhäuser, Boston (1999)

2. Crewe, A.V., Crewe, D.A.: Inexact Reconstruction: Some Improvements. Ultramicroscopy 16 (1985) 33-40

3. Gordon, R., Herman, G.T.: Reconstruction of Pictures from their Projections. Graphics Image Process. 14 (1971) 759-768

4. Kuba, A.: The Reconstruction of Two-Directionally Connected Binary Patterns from their Two Orthogonal Projections. Comp. Vision, Graphics, and Image Proc. 27 (1984) 249-265

5. Prause, G.M.P., Onnasch, D.G.W.: Binary Reconstruction of the Heart Chambers from Biplane Angiographic Image Sequences. IEEE Trans. Medical Imaging MI-15 (1996) 532-546

6. Schilferstein, A.R., Chien, Y.T.: Switching Components and the Ambiguity Problem in the Reconstruction of Pictures from their Projections. Pattern Recognition 10 (1978) 327-340

7. Balázs, P., Balogh, E., Kuba, A.: Reconstruction of 8-Connected but not 4Connected $h v$-Convex Discrete Sets. Discrete App. Math. 147 (2005) 149-168

8. Del Lungo, A.: Polyominoes Defined by Two Vectors. Theor. Comput. Sci. 127 (1994) 187-198

9. Kuba, A., Balogh, E.: Reconstruction of Convex 2D Discrete Sets in Polynomial Time. Theor. Comput. Sci. 283 (2002) 223-242

10. Barcucci, E., Del Lungo, A., Nivat, M., Pinzani, R.: Reconstructing Convex Polyominoes from Horizontal and Vertical Projections. Theor. Comput. Sci. 155 (1996) 321-347

11. Del Lungo, A., Nivat, M., Pinzani, R.: The Number of Convex Polyominoes Recostructible from their Orthogonal Projections. Discrete Math. 157 (1996) 65-78

12. Feretic, S., Svrtan, D.: Combinatorics of Diagonally Convex Directed Polyominoes, Discrete Math. 157 (1996) 147-168

13. Golomb, S.W.: Polyominoes. Charles Scriber's Sons, New York (1965)

14. Balázs, P.: A Decomposition Technique for Reconstructing Discrete Sets from Four Projections. Image and Vision Computing, submitted

15. Ryser, H.J.: Combinatorial Mathematics. The Carus Mathematical Monographs $14(1963)$ 